\title{
Optical PT-Symmetric Counterparts of Ordinary Metals
}

\author{
Constantinos A. Valagiannopoulos \\ Department of Physics, School of Science and Technology, \\ Nazarbayev University, 53 Qabanbay Batyr Ave, Astana, KZ-010000, Kazakhstan \\ Email: konstantinos.valagiannopoulos@nu.edu.kz
}

\begin{abstract}
How one can fabricate the Parity-Time (PT) symmetric siblings of commonly used metals in the visible? This work tries to give an answer to that question by providing the features of the active media which conjugately pair the complex permittivities of ordinary metals (Copper, Aluminum, Silver, Gold, Platinum) at the optical frequencies. The frequency response model of quantum dots (QDs) is used to evaluate the effective permittivity of the active material; their characteristics which give a PT-symmetric counterpart for the considered metal are deduced through a multi-step optimization process. The required resonance frequencies, loss factors and degrees of population inversion for the QDs are provided for various frequencies and metals. The response of the metals when they are PT-symmetrically coupled with the provided mixtures is demonstrated in specific photonic configurations and interesting properties with certain applicability potential are revealed.
\end{abstract}

Metal, Parity-Time (PT) Symmetry, Quantum Dot (QD), Visible Spectrum.

\section{INTRODUCTION}

Parity-Time (PT) symmetry, a concept originating from Quantum Mechanics, is a property attributed to systems having real eigenvalues even though their Hamiltonian matrix is nonHermitian [1]. If one takes a $2 \times 2$ matrix with frequencydependent elements, one can directly understand that there are combinations of complex diagonal elements which lead to real eigenvalues; when the frequency (or another parameter) changes and this condition is no more valid, then we say that we have a PT symmetry breaking [2]. If the aforementioned PT symmetry concept gets particularized to Electromagnetics, it yields to material distributions whose complex (due to harmonic time dependence) permittivities and permeabilities are conjugately symmetric around a center or plane of symmetry. In other words, the lossy and active parts are balanced both in terms of their magnitudes and their positions which confers the system an overall loss-free behavior. Such a balanced combination of materials may be used as a rule to restrict the multi-dimensional parametric space for electromagnetic systems and converge to optimized designs in a more direct way.

Numerous functional electromagnetic devices have been modeled and designed in this way, from deeply subwavelength waveguides with asymmetric optical propagation [3] and modulators [4] with complete control over amplification and absorption to metasurfaces comprised of artificial metaatoms [5] offering a huge number of degrees of freedom to the designer. Furthermore, PT-symmetric wave steering components [6], incorporating plasmonic media, have been analyzed and certain designs have been proposed [7] which exploit inverse-rib waveguides. The concept of PT symmetry has been also experimentally demonstrated in large-scale temporal photonic lattices where light transport is performed very successfully [8]. Finally, highly efficient cloaks [9], perfect planar lenses [10] in the geometrical optics sense and general field transformers [11] have been proposed based on and inspired by the property of PT symmetry.

Outside the PT symmetry framework, combining passive materials (loads) and especially metals with active components (sources) has been a routine procedure for very many decades in designing microwave and photonic (electromagnetic in general) components. If one gets focused to recent and modern ideas, one can certainly include fishnet structures comprised of metals and epoxy doped with Rhodamine dye in order to fabricate lossless double negative media [12] and combinations of silver plasmonic shells with rare-earth ions to constitute coated nanoparticle lasers [13]. In addition, dye active molecules have been also employed to compensate the losses in planar metallic structures [14] and nanocomposite multilayers [15]. Another significant category of media with active behavior host quantum dots (QDs), namely semiconductor particles which are efficient photon emitters when properly excited. In particular, bi-dimensional arrays of colloidal QDs (belonging to the groups II-VI of the periodic table of the elements) can be combined with nonlinear media to elaborate devices with memory [16], while a deposition of QDs on top of Polyallylamine Hydrochloride layer and sandwiched in a metal nanostructure has been reported to increase dramatically the speed of spontaneous emission [17]. Quantum dots of Indium Gallium Arsenide near a silver interface can also substantially enhance electric and magnetic vacuum fluctuations [18].

In this work, the aforementioned two topics (PT-symmetry, metals) are combined: we are using techniques that confer active behavior to materials which are paired in a PTsymmetric way with common metals at the optical frequency range. In particular, we perform a multi-step optimization to determine the characteristics of the host materials and the proper dose and texture of quantum dots that constitute the PTsymmetric counterparts of bulk metals (materials with complex conjugate permittivities each other) at every single operating frequency. The complex dielectric constants of metals are obtained through measurements [19] compiled by commonly used databases [20]. The effective complex permittivity of 
the active medium, namely the host material and the QDs, is evaluated through Lorentz-Drude model in frequency domain followed by implementation of Maxwell-Garnet mixing formula [21]. The characteristic parameters of the quantum dots are found and represented when the systems are excited by visible light. In this way, we obtain the features of QDs which, dispersed into a suitable host material, work as PTsymmetric siblings for ordinary metals (Copper, Aluminum, Silver, Gold, Platinum). The derived PT-symmetric couples are used in two specific components (bi-layer and touching cylinders) whose fabrication is assisted by metals nature, and are found to possess certain interesting properties beneficial for light steering and photonic energy accumulation.

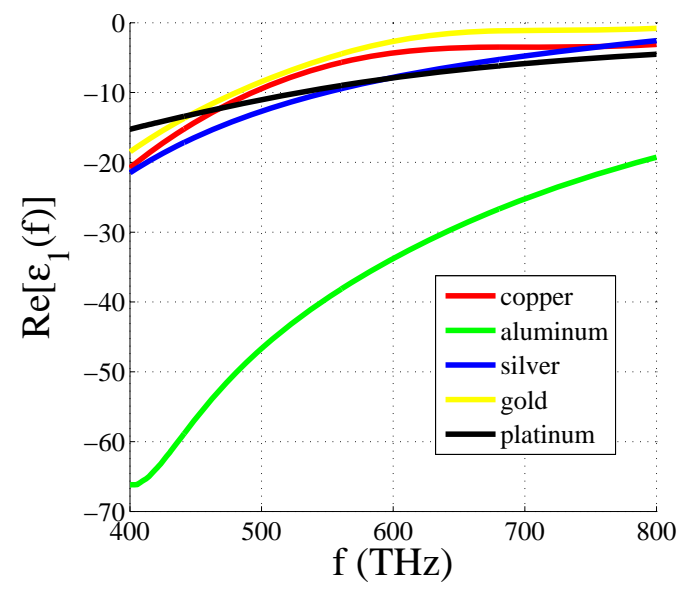

(a)

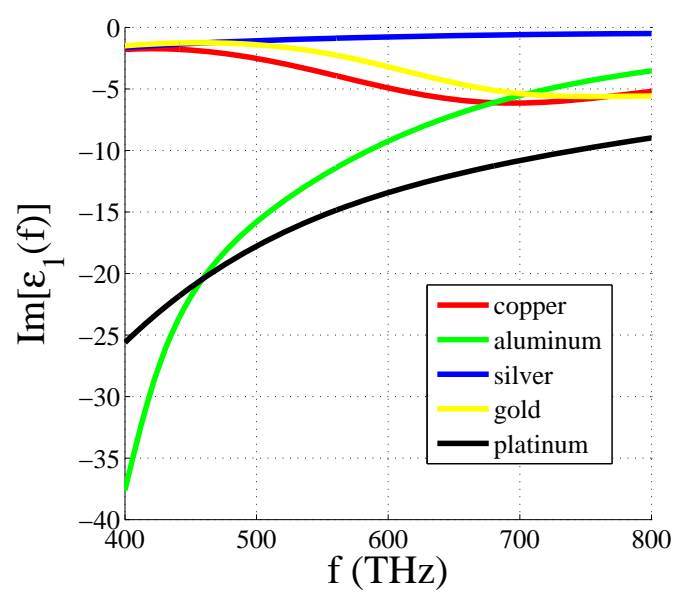

(b)

Fig. 1. The: (a) real and (b) imaginary relative permittivity $\varepsilon_{1}(f)$ for five different metals in the visible spectrum $(400 \mathrm{THz}<f<800 \mathrm{THz})$.

\section{Complex Permittivities}

\section{A. The Metals}

We consider five of the most commonly used metals in photonic applications: noble (Silver, Gold, Platinum) or not (Copper, Aluminum). We utilize one of the most valid set of measurements [19] which is hosted by one of the most wellestablished material databases [20]. The provided analysis is confined to the optical frequencies: $400 \mathrm{THz}<f<800 \mathrm{THz}$ and the suppressed harmonic time dependence is of the form: $e^{+j 2 \pi f t}$. In Figs. 1, we show the real (Fig. 1(a)) and the imaginary (Fig. 1(b)) parts of the relative permittivities $\varepsilon_{1}$ of the five metals, as functions of frequency $f$. Due to the adopted time dependence, the imaginary parts of $\varepsilon_{1}(f)$ in lossy media (like the considered metals) are negative $\left(\operatorname{Im}\left[\varepsilon_{1}(f)\right]<0\right)$. As it is obvious from Fig. 1(a), all the metals across the regarded frequency range exhibit strong plasmonic response and thus $\operatorname{Re}\left[\varepsilon_{1}(f)\right]<0$ (such a feature becomes very striking in the case of aluminum). In particular, the real parts become less negative as the operational frequency increases. As far as the imaginary parts are concerned, aluminum and platinum are very lossy and get less lossy for increasing $f$, while the overall passivity of silver is small compared to all the other ones. Gold and copper have relatively small losses for smaller frequencies (red, orange, yellow colored light) which are increased for larger frequencies (green, blue, violet colored light).

\section{B. The Active Media}

When it comes to the active material with relative dielectric constant $\varepsilon_{r 2}$, we use an effective model which has been thoroughly described [21] and extensively utilized [22], [23], [24]. More specifically, the relative permittivity of the quantum dots $\varepsilon_{Q D}(f)$ follows a Drude-Lorentz model written as:

$$
\varepsilon_{Q D}(f)=\varepsilon_{\text {host }}\left[1+\frac{6\left(2 a^{2}-1\right) f_{P}}{\pi^{2}\left(f_{R}-f+j \Gamma\right)}\right] .
$$

Obviously, the symbol $\varepsilon_{\text {host }}$ denotes the relative dielectric constant of the host material which affects the behavior of the quantum dots. It should be stressed that (1) is not the effective permittivity $\varepsilon_{2}(f)$ of the mixture of QDs into the host medium but corresponds to the dielectric behavior of the particles themselves which are strongly influenced by their environment. We assume a dispersionless and lossless host material $\left(\operatorname{Im}\left[\varepsilon_{\text {host }}\right]=0\right)$ with permittivity within the value range: $1<\varepsilon_{\text {host }}<15$ which corresponds to a variety of different media (from polymers to semiconductors) at the optical frequencies.

The parameter $0<a<1$ is a probability describing how hard is for the QDs to get activated and abandon their ground state to populate the exciton states. For $a=1$ we do not have any reaction from the side of QDs and for $a=0$ all the quantum dots are moving to exciton states and energize the medium. The amplitude $a$ is taken here as a constant representing the average state of a quantum dot ensemble (microcavities, II-VI elements, single-exciton compounds) and is smaller for more active media. To put it alternatively, the parameter $a$ is inversely related to the population exchange between the conduction and valence bands of the effective medium since it equals to the average occupation factor of the ground state [25]. Note that $a^{2}>1 / 2$ indicates an optical loss and $a^{2}<1 / 2$ an optical gain for the background electric field; therefore, in our studies (since we aim at $\operatorname{Im}\left[\varepsilon_{2}(f)\right]>0$ ) only cases with $0<a^{2}<1 / 2$ are regarded. The parameter $f_{R}$ is the resonance frequency at which the imaginary part $\operatorname{Im}\left[\varepsilon_{Q D}\right]$ gets maximized (in absolute values). In the vicinity 
of $f=f_{R}$, the real part $\operatorname{Re}\left[\varepsilon_{Q D}\right]$ makes a sharp variation following the shape of return-to-zero pulse around $\varepsilon_{\text {host }}$ as dictated by the corresponding Kramers-Kronig relation [26]: $\operatorname{Re}\left[\varepsilon_{Q D}(f)\right]=1+\frac{2}{\pi} \int_{0}^{+\infty} \frac{\operatorname{Im}\left[\varepsilon_{Q D}(\varphi)\right] \varphi}{\varphi^{2}-f^{2}} d \varphi$. The parameter $\Gamma$ is labeled as "loss factor" (since it is multiplied by the imaginary unit $j$ in (1)) and is expressed in frequency units; it determines how abrupt is the resonance, namely how narrow would be the frequency interval at which the change in $\varepsilon_{Q D}(f)$ happens. Finally, let us conveniently call the parameter $f_{P}$ a "plasma" frequency which, as it is obvious from (1), makes stronger the frequency-dependent part of the permittivity and therefore enhances the resonance (smaller $\Gamma$, larger maxima for $\operatorname{Im}\left[\varepsilon_{Q D}(f)\right]$ and more abrupt variations for $\operatorname{Re}\left[\varepsilon_{Q D}(f)\right]$ close to $f=f_{R}$ ). One can point out alternatively that $f_{P}$ corresponds to a cross section of QD activity. A typical choice is to assume loss factors and plasma frequencies much smaller than the resonance frequency: $\Gamma, f_{P} \ll f_{R}$.

The quantum dots described by (1), whose parameters have been defined and described above, are hosted by a background medium with relative dielectric constant $\varepsilon_{\text {host }}$ as already mentioned. If one implements the Maxwell-Garnett mixing formula [21], [27] which takes into account the shape of the inclusions: $\frac{\varepsilon_{2}(f)-\varepsilon_{\text {host }}}{\varepsilon_{2}(f)+2 \varepsilon_{\text {host }}}=s \frac{\varepsilon_{Q D}(f)-\varepsilon_{\text {host }}}{\varepsilon_{Q D}(f)+2 \varepsilon_{\text {host }}}$, one obtains:

$$
\varepsilon_{2}(f)=\varepsilon_{\text {host }} \frac{2\left(1-2 a^{2}\right)(1+2 s) f_{P}+\pi^{2}\left(f-f_{R}-j \Gamma\right)}{2\left(1-2 a^{2}\right)(1-s) f_{P}+\pi^{2}\left(f-f_{R}-j \Gamma\right)} .
$$

The ratio $s$ is the filling factor of the quantum dots, namely the percentage of the volume of the host medium that is occupied by QDs. It can be checked that if $s=0 \%$ the material is the same with the host: $\varepsilon_{2}(f)=\varepsilon_{\text {host }}$. It should be stressed that one could use the Bruggeman's model [28] for averaging instead of the aforementioned homogenization formula; however, we prefer to work with permittivities rather than employing conductivities in our study.

\section{PT-SYMMETRIC PAIRS}

\section{A. Parameters Variations and Description}

The major purpose of this work is to provide the designer with two sets of parameters $\left(f_{R}, \Gamma, f_{P}\right)$ and $(a, s)$ determining the quantum dots which hosted by a material with permittivity $\varepsilon_{\text {host }}$, lead to an effective medium $\varepsilon_{2}(f)$ behaving as the PT counterpart of a specific metal (with dielectric constant $\varepsilon_{1}(f)$ ) at a specific frequency $f$ (namely: $\varepsilon_{2}(f)=\varepsilon_{1}^{*}(f)$ ). In order to retrieve this data, we perform a step-by-step adaptive optimization (with greedy approach at each step) to achieve $\varepsilon_{2}(f)=\varepsilon_{1}^{*}(f)$ for each of the five metals (Copper, Aluminum, Silver, Gold, Platinum) and for every single frequency $f$, with respect to the six parameters $\left\{f_{R}, \Gamma, f_{P}, a, s, \varepsilon_{\text {host }}\right\}$ incorporated in (2). In particular, we are searching for the optimal resonance frequency $f_{R}$ close to the current frequency $f$ since we need the sharp variations of $\varepsilon_{Q D}(f)$ close to the frequency $f$ under investigation, namely: $f<f_{R}<1.1 f$. We choose $f_{R}>f$ for reasons that will become obvious in the following. As far as the frequencies $\left(\Gamma, f_{P}\right)$ are concerned, they are sought within a range like: $0.001 f<\Gamma, f_{P}<0.2 f$. We cannot admit $\Gamma=0$ since: (i) the losses are inevitable and

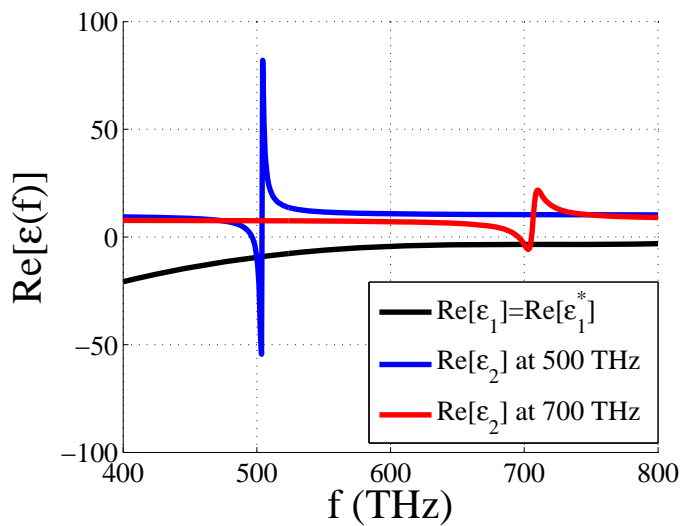

(a)

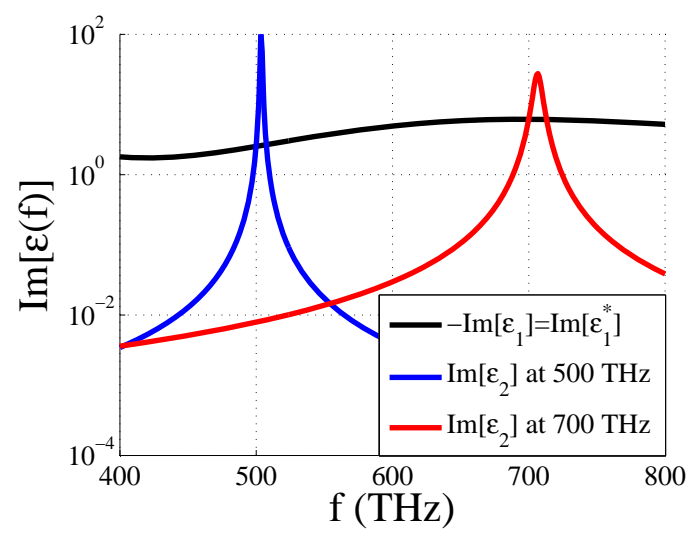

(b)

Fig. 2. The: (a) real and (b) imaginary parts of the permittivities of copper $\left(\varepsilon_{1}(f)\right)$ and the two active materials $\left(\varepsilon_{2}(f)\right)$ that mimic the PT-symmetric counterpart of copper at $f=500 \mathrm{THz}$ and $f=700 \mathrm{THz}$, as functions of operating frequency $f$.

(ii) if so, the problem becomes trivial. To put it alternatively, if $\Gamma=0$, both the real and the imaginary part of $\varepsilon_{2}(f)$ tends to infinity for $f \rightarrow f_{R}\left(\operatorname{Re}\left[\varepsilon_{2}\right]\right.$ jumps from $-\infty$ to $+\infty$, while $\operatorname{Im}\left[\varepsilon_{2}\right]$ increases abruptly from a small positive value to $+\infty$ ); thus, every possible value of $\varepsilon_{1}^{*}$ is crossed by the infinite jumps of $\varepsilon_{2}(f)$ in the vicinity of $f=f_{R}$. When it comes to the host permittivity $\varepsilon_{\text {host }}$ and the ground state amplitude $a$, they can vary within the aforementioned intervals: $1<\varepsilon_{\text {host }}<15$ and $0<a^{2}<0.5$. The filling factor $s$ cannot be very close to unity otherwise the adopted Maxwell-Garnett approximation is not valid; thus we assume: $0 \%<s<50 \%$.

The intervals of the input parameters defined above concern only the first (initial) step of the greedy optimization. At every single step the ranges become smaller based on the variations of the previous step with the same number of points. In this way, we trap the optimal solutions within shorter and shorter value intervals which are more and more finely chopped. If the best results are appeared on the upper or lower limits of the initial ranges, we moderately expand them. We do not claim that the followed optimization process is the best for that kind of objective function defined as the normalized relative difference between the permittivity $\varepsilon_{2}$ and the ideal PT-symmetric value $\varepsilon_{1}^{*}: \frac{\left|\varepsilon_{2}-\varepsilon_{1}^{*}\right|}{\left|\varepsilon_{2}\right|+\left|\varepsilon_{1}^{*}\right|}$ (with optimization 
parameters $\left\{f_{R}, \Gamma, f_{P}, a, s, \varepsilon_{\text {host }}\right\}$, constant $f$ and specific kind of metal). However, the average error that we obtain (value of the objective function referred above) gets as low as $0.005 \%$ without demanding high computational power. It is therefore sensible to assume that with use of more advanced computational grids and by adopting smarter optimization techniques, one can achieve perfect PT symmetry for the considered metals at particular frequencies.

The graphs from a typical/indicative execution of the aforementioned optimization procedure are depicted in Figs. 2. More specifically, we show the frequency-dependent variations of the real (Fig. 2(a)) and the imaginary (Fig. 2(b)) parts of the complex permittivities $\varepsilon_{1}=\varepsilon_{1}(f)$ of an ordinary metal (copper in this particular case). We also illustrate the dielectric behavior of the corresponding mixtures of quantum dots into a host medium when they are approximating PT symmetry at $f=500 \mathrm{THz}$ (blue curve, orange light) and at $f=700$ $\mathrm{THz}$ (red curve, violet light). It is clear that the conjugate approximation $\left(\varepsilon_{2} \cong \varepsilon_{1}^{*}\right)$ dictated by $\mathrm{PT}$ symmetry is well achieved in the vicinity of the two considered frequencies by the two active materials. This is the outcome of the resonances which are occurring very close to the two selected frequencies $\left(f_{R}=510 \mathrm{THz}\right.$ for $f=500 \mathrm{THz}$ and $f_{R}=714 \mathrm{THz}$ for $f=700 \mathrm{THz}$ ). This substantial change in value happening at a resonance makes the frequency-resonating quantity (permittivity in our case) to cover a huge range of values within a very small interval of frequencies; therefore, one can calibrate the rest of the model's parameters to achieve an exact equality of the quantity $\left(\varepsilon_{2}(f)\right)$ with the given value $\left(\varepsilon_{1}^{*}(f)\right)$ at the specific frequency $f$. The significant change in values of $\operatorname{Im}\left[\varepsilon_{2}(f)\right]$ may raise certain concerns related to the overall character (passive or active) of the structure. If it operates at a slightly higher frequency than the selected $f$, the imaginary part of the active counterpart would be much larger than that dictated by PT-symmetry; therefore, the operational frequency should be carefully chosen otherwise the system would act as a laser and lose its PT-symmetric (lossless) response.

It is due to this calibration that the resonance of the second active material (at $f=700 \mathrm{THz}$ ) is broader $(\Gamma=0.005 f$ and $f_{P}=0.084 f$ ) than the resonance of the first material at $f=500 \mathrm{THz}$, where: $\Gamma=0.001 f$ and $f_{P}=0.09 f$. It is noted that since the real part of dielectric constants $\varepsilon_{2}$ should always be negative (due to the plasmonic nature of metals), the resonance frequency is always greater than the frequency $f$ of PT-symmetry. That is because the equality $\varepsilon_{2}=\varepsilon_{1}^{*}$ is feasible only across the decreasing negative part of the variation $\operatorname{Re}\left[\varepsilon_{2}(f)\right]$ which corresponds to $f<f_{R}$ (see Fig. 2(a)). Additionally, the quantum dots of the first active mixture (activated at $f=500 \mathrm{THz}$ ) are getting equally easily energized ( $a=0.14$, i.e. closer to full inversion ) but occupy much less space $(s=28 \%)$ than the corresponding QDs of the mixture activated at $f=700 \mathrm{THz}(s=35 \%)$. As far as the permittivities of the host medium are concerned, they are quite high for both materials: $\varepsilon_{\text {host }}=10$ for the first and $\varepsilon_{\text {host }}=8$ for the second one, which can be searched within the family of optical semiconductors [21], [29], [30], [31], [32].

As far as the stability of the system is concerned, it is definitely an issue to be considered since the system involves active elements. In [9], a related system was studied for different purposes (cloaks modeling), and it was found that it is always possible to design a specific dispersion of the active and passive elements that guarantee full stability. For this reason, we expect that, also for the structures involving the aforementioned active media as PT symmetric twins of ordinary metals, full stability for any finite input signal can be achieved. The demand for stable system could play the role of an extra constraint in the optimization formulation, which would be the topic of a future work.

The results of the optimization technique described in the previous paragraphs which correspond to PT-symmetric relations as these indicated by Figs. 2, are presented in the following. We think that there is not a contradiction between large number (six) of input parameters $\left\{f_{R}, \Gamma, f_{P}, a, s, \varepsilon_{\text {host }}\right\}$ which are swept in order to satisfy one complex condition: $\varepsilon_{2}(f)=\varepsilon_{1}^{*}(f)$. If the number of input parameters is the same with the number of minimized objectives (deviations from exact equalities), this does not necessarily guarantee exact equalities unless we consider linear objectives (and even in this case, not always). Furthermore, having many degrees of freedom gives the designer the flexibility to conclude to alternative solutions or introducing constraints related to the input parameters (such as a high $a$ in our case).

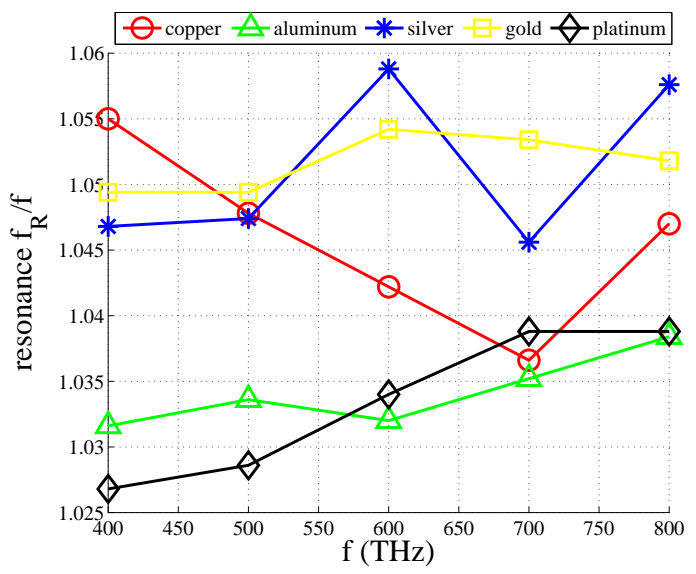

Fig. 3. The necessary (normalized) resonant frequencies $f_{R} / f$ of the QDs mixture to constitute a PT-symmetric medium for each metal at the considered frequency $f$.

\section{B. Main Results and Discussion}

In Fig. 3, we show the (normalized) optimal resonance frequencies $f_{R} / f$ of the QDs which are required in order to build a PT-symmetric counterpart of the corresponding metal (Copper, Aluminum, Silver, Gold, Platinum) as function of operating frequency $f$. As indicated above, the resonance frequency $f_{R}$ is always larger than $f$ because only then the variation of $\operatorname{Re}\left[\varepsilon_{2}(f)\right]$ can take the necessary negative value $\operatorname{Re}\left[\varepsilon_{1}^{*}(f)\right]$. Tuning the resonance frequency of the quantum dots to a specific value is not a trivial procedure. However, certain methods that can control the frequency response of QDs have been reported [33], [34] and implemented [35].

In Fig. 4, the (normalized) optimal factors $\Gamma / f$ determining the losses in the corresponding QDs mixtures are shown for 


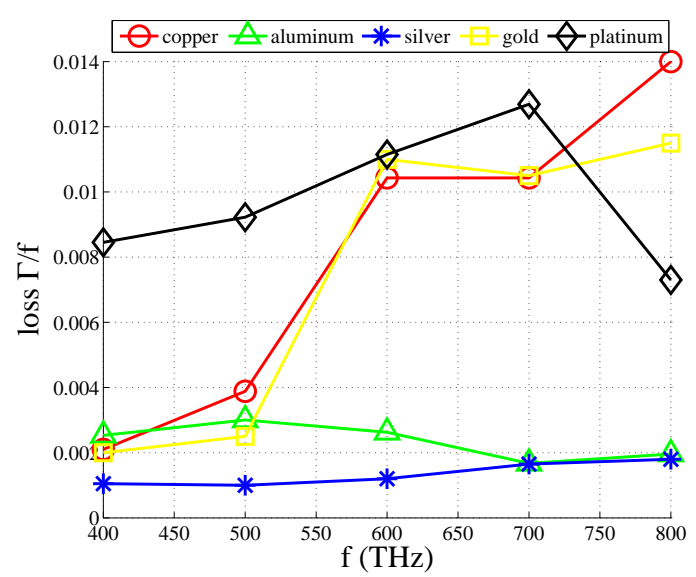

Fig. 4. The necessary (normalized) loss factors $\Gamma / f$ of the QDs mixture to constitute a PT-symmetric medium for each metal at the considered frequency $f$.

various frequencies and metals. Very low-loss quantum dots are employed regardless of the considered metal. Especially for silver and aluminum the parameter $\Gamma$ is extremely small in the visible spectrum; such a feature is attributed to the very small $\left|\operatorname{Im}\left[\varepsilon_{1}\right]\right|$ for silver and the very negative $\operatorname{Re}\left[\varepsilon_{1}\right]$ and $\operatorname{Im}\left[\varepsilon_{1}\right]$ simultaneously for aluminum. On the contrary, the loss tolerance gets higher for copper and gold as the frequency $f$ increases and is overall substantial for the case of platinum. Despite the fact that locating materials to create quantum dots of small losses is a quite challenging task for, specific techniques have been successfully utilized [36], [37].

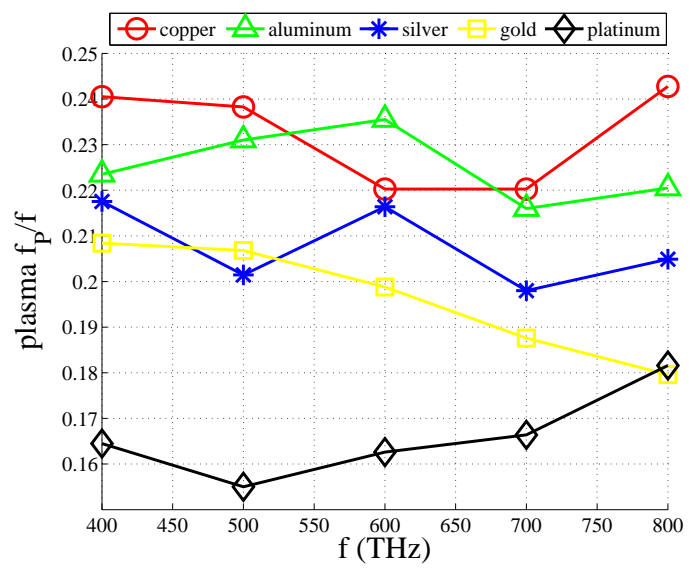

Fig. 5. The necessary (normalized) "plasma" frequencies $f_{P} / f$ (or cross sections) of the QDs mixture to constitute a PT-symmetric medium for each metal at the considered frequency $f$.

In Fig. 5, we represent the (normalized) optimal plasma frequencies $f_{P} / f$ which should characterize QDs in order to make a PT-symmetric pair for the regarded metals and frequencies. This is not a very crucial quantity since its role can get easily compensated by the loss factor $\Gamma$. As far as the variations are concerned, we observe that PT-pair of the platinum possesses the lowest $f_{P}$ which is due to the relatively flat spectrum of its real permittivity as indicated by Fig. 1(a). On the other hand, the higher $f_{P}$ is needed in the cases of copper and aluminum, while gold and silver require moderate plasma frequencies for their PT-symmetric siblings which on average decrease with respect to $f$.

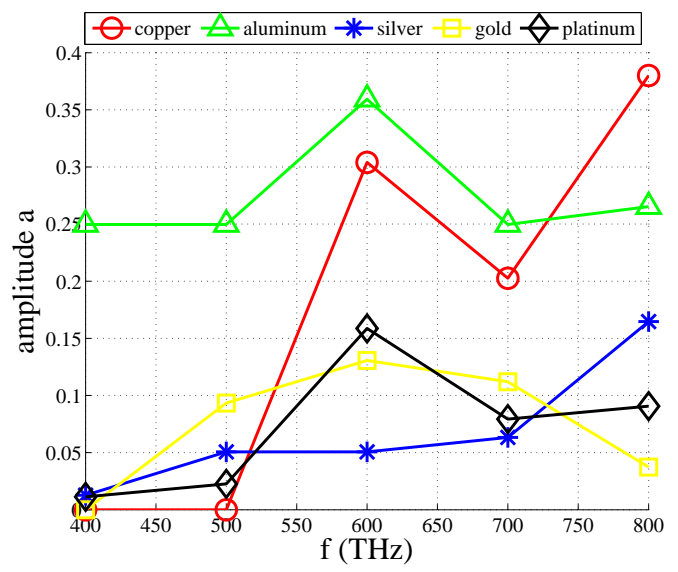

Fig. 6. The amplitude associated to ground-state occupation $a$ of the QDs whose mixture (with a host material) constitutes a PT-symmetric medium for each metal at the considered frequency $f$.

In Fig. 6, the necessary amplitudes associated to groundstate occupation of the QDs $a$ is depicted as function of the operating frequency for all the considered cases. It is remarkable that aluminum, despite the its huge losses (see Fig. 1(b)), requires modestly inversed (small gain, large a) quantum dots for its PT-symmetric pair, a feature that is related to the very small losses of the corresponding mixture (see Fig. 4). All the noble metals (and copper) demand very small $a$ for lower frequencies but such a request gets relaxed for increasing $f$. When it comes to gold, it requires QDs mixtures with small $a$ both at low (red light radiation) and high (violet light radiation) frequencies and can tolerate larger amplitudes in-between (green light radiation). Selecting the amplitude $a$ of QDs at a given frequency is doable; in patricular, there are plenty of active (emissive) elements and compounds which can play the role of quantum dots and additionally certain controlling processes are described in the bibliography [38], [39], [40], [41].

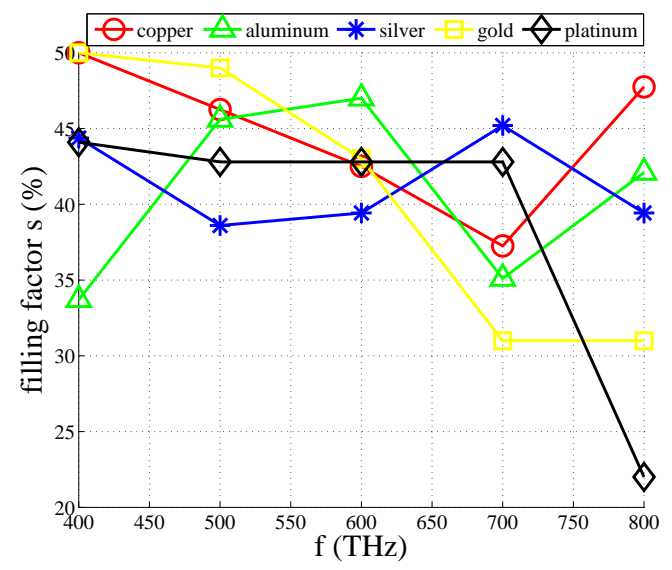

Fig. 7. The volume over volume concentration $s$ (in percentage) of QDs whose mixture (with a host material) constitutes a PT-symmetric medium for each metal at the considered frequency $f$.

In. Fig. 7, we show the variation of the optimal filling 
factor $s=s(f)$ (in volume over volume percentage) of the quantum dots into the host material, a mixture which describes the active medium, namely the PT-symmetric twin of the regarded metal at frequency $f$. It is clear that PT-symmetry does not demand very large concentration of quantum dots into the host substance otherwise Maxwell-Garnett approximation would not be valid. Most importantly, there are cases that the necessary volume over volume ratio $s$ falls below $35 \%$ which means that the mixture can work as a well homogenized medium. Gold needs less and less QDs to equalize their losses as $f$ goes toward ultraviolet (UV), while silver, aluminum, copper and platinum (far from the violet spectrum) exhibit a relative stability in terms of the required $s$.

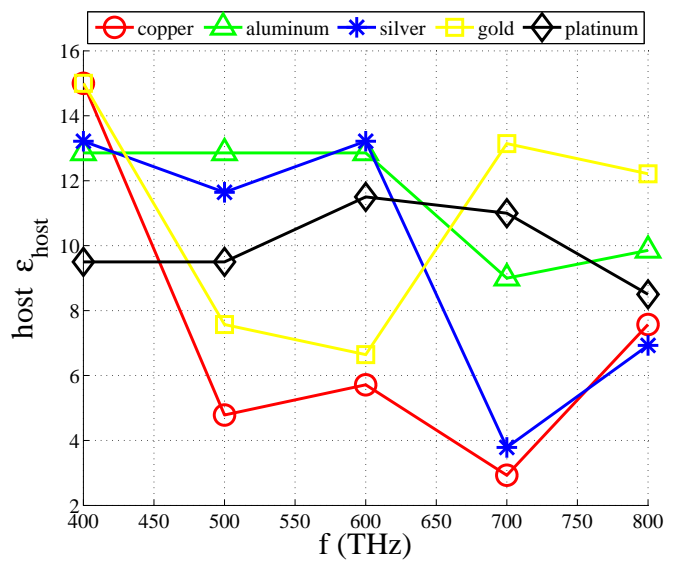

Fig. 8. The necessary (lossless) relative permittivity $\varepsilon_{\text {host }}$ of the medium that hosts the QDs so that the overall mixture to constitute a PT-symmetric medium for each metal at the considered frequency $f$.

In Fig. 8, we represent the permittivity $\varepsilon_{\text {host }}=\varepsilon_{\text {host }}(f)$ that the (lossless) host material of the PT-symmetric medium should have for every single of the considered metals and $f$. We note once again that the flattest curves are obtained for aluminum and platinum, while silver and copper need much different hosts for different frequencies.

It should be remarked that this study gives only the optimal set of parameters $\left\{f_{R}, \Gamma, f_{P}, a, s, \varepsilon_{h o s t}\right\}$ that make $\varepsilon_{2}(f)=\varepsilon_{1}^{*}(f)$ and does not examine if such combinations of resonance frequencies $\left(f_{R}\right)$, loss factors $\Gamma$, plasma frequencies $f_{P}$, ground-state occupation degrees $a$, filling factors $s$ and host permittivities $\varepsilon_{\text {host }}$ are feasible at the specific operating frequency $f$. Finding and fabricating actual active materials that can achieve PT-symmetry for the commonly used metals is a very ambitious target that is not addressed in the work at hand. However, the presented results can be very useful for the device designer since one can estimate how different are the available combinations for QDs $\left(f_{R}, \Gamma, f_{P}, a, s\right)$ and hosts materials $\left(\varepsilon_{\text {host }}\right)$ from the ideal combinations given by the graphs in Figs. 3-8.

Most importantly, the adopted process can be modified (and that would be the objective of a future study) to return not one, but multiple solution sets $\left\{f_{R}, \Gamma, f_{P}, a, s, \varepsilon_{\text {host }}\right\}$ which correspond to an overall good performance $\left(\varepsilon_{2} \cong \varepsilon_{1}^{*}\right)$. In this way, one can relax the criterion of exact PT-symmetry and select that solution which obeys better the availabil- ity/cost constraints and the feasibility/integration limitations. For example in Figs. 2, the presented variations of $\varepsilon_{2}(f)$ that makes a PT-symmetric pair for copper at $f=500 \mathrm{THz}$ and $f=700 \mathrm{THz}$ are different from those reported as optimal in Figs. 3-8 (copper, red curves). This is an indication that there are multiple feasible solutions to design PT-symmetric active media for particular metals and frequencies. An additional challenge would be not to find PT-symmetric pairs for one frequency $f$ but for a specific spectral range. That could be also feasible if we relax further our demands for perfect PT symmetry and employ higher computing power.

An objection that could be also raised regarding the presented study is to what extent the adopted classical model (1), which ignores saturation and quantum phenomena, can describe combinations of quantum dots. One can respond that if the considered time frames are small enough (which may be possible since we are referring to optical frequencies), the active behaviors predicted by the classical model can approximate relatively well the reality.

\section{Specific Configurations}

Based on the remarks above, we assume that one can find, at an optical frequency $f$, a suitable distribution of QDs with characteristics $\left(f_{R}, \Gamma, f_{P}, a\right)$ occupying a portion $s$ of the volume of a host medium with permittivity $\varepsilon_{\text {host }}$, which gives a dielectric response very close to the PT-symmetric one of the considered metal. Therefore, we can proceed to the demonstration of the response of PT-symmetric couple in configurations that the role of passive electrode can be played well by ordinary metals. In the following, we use the notation $k_{0}=2 \pi f \sqrt{\varepsilon_{0} \mu_{0}}=2 \pi / \lambda_{0}$ for the operational wavenumber into vacuum ( $\lambda_{0}$ is the operational wavelength into vacuum).

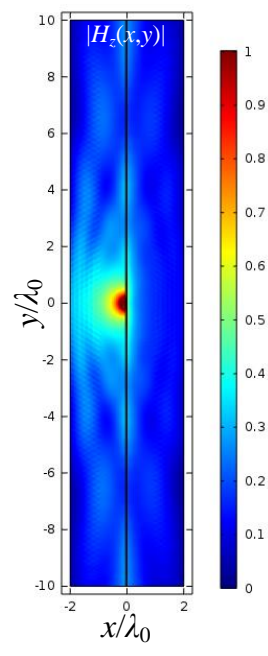

(a)

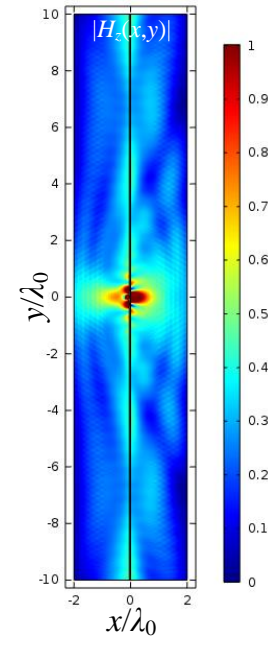

(b)

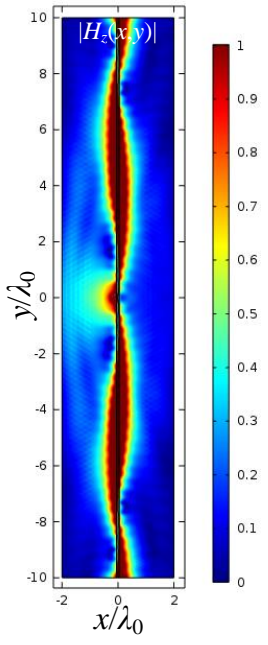

(c)
Fig. 9. The spatial distribution of the magnitude of the magnetic field $\left|H_{z}(x, y)\right|$ on the $x y$ plane when a $\hat{\mathbf{z}}$-polarized magnetic current is illuminating at distance $w=0.05 \lambda_{0}$ from: (a) a slab of metal with thickness $d$, (b) a slab of the PT-symmetric pair of the metal with thickness $d$, (c) two PT-symmetric slabs back-to-back each one with thickness $d$. Plot parameters: copper, $f=600 \mathrm{THz}, \varepsilon_{1} \cong-4.3-4.9 j, d=0.05 \lambda_{0}$. 


\section{A. The Case of Bi-layer}

Since metals can easily be shaped into thin planar sheets and active QD-enriched sheets can be deposited on them, the configuration of a bi-layer [42], [43], [44] (two successive layers of the same thickness $d$ ) fits well within our considerations. Therefore, we consider two layers positioned backto-back parallel to $y z$ plane (normal to the $\hat{\mathbf{x}}$ axis): one made of the considered metal of dielectric constant $\varepsilon_{1}$ and another made of the corresponding mixture (with dielectric constant $\varepsilon_{2}=\varepsilon_{1}^{*}$ ) of quantum dots (permittivity $\varepsilon_{Q D}$ ) hosted in a suitable background medium (permittivity $\varepsilon_{\text {host }}$ ). To obey the PT-symmetry, the layers have equal thickness $d$ and if one assumes electrically small thicknesses, namely $k_{0} d \ll 1$, one obtains the following approximate formulas (via Taylor expansion) for the reflection and transmission coefficients respectively:

$$
\begin{array}{r}
\rho=\frac{k_{0} d}{2 \sqrt{u^{2}-1}} . \\
{\left[u^{2}\left(\varepsilon_{1}+\varepsilon_{2}-\frac{\varepsilon_{1}+\varepsilon_{2}}{\varepsilon_{1} \varepsilon_{2}}\right)+2-\varepsilon_{1}-\varepsilon_{2}\right],} \\
\tau=1-\frac{k_{0} d}{2 \sqrt{u^{2}-1}} . \\
{\left[u^{2}\left(\varepsilon_{1}+\varepsilon_{2}+\frac{\varepsilon_{1}+\varepsilon_{2}}{\varepsilon_{1} \varepsilon_{2}}-4\right)+2-\varepsilon_{1}-\varepsilon_{2}\right] .}
\end{array}
$$

The notation $u=k_{t} / k_{0}$ is used for the relative transverse wavenumber that distinguishes the propagating $(|u|<1)$ from the evanescent [45] $(|u|>1)$ waves.

The condition to have real reflection and transmission coefficients for evanescent waves is to have PT-symmetry with respect to the common surface of the two slabs. If $\rho, \tau \in \mathbb{R}$, the magnitude of the evanescent waves along the common boundary gets conserved and the overall field concentration becomes enhanced [46]. In order to feed the structure with evanescent waves, we use a magnetic current line source located very close to the slab surface (at distance $w=0.05 \lambda_{0}$ ). As far as the metal is concerned, we choose copper working at the green part of the visible spectrum $(f=600 \mathrm{THz})$ at which $\varepsilon_{1} \cong-4.3-4.9 j$. For its PT-symmetric twin we need, according to Figs. 3-8, a lossless medium [31], [32] with $\varepsilon_{\text {host }} \cong 6$ which hosts quantum dots with moderate loss factor $\Gamma \cong 0.01 f$ and plasma frequency $f_{P} \cong 0.22 f$ [29], while possessing a substantial ground state occupation corresponding to $a \cong 0.3$. The QDs should occupy $s \cong 40 \%$ of the volume of the host material a dose with which the Maxwell-Garnett formula [27] is valid.

In Figs. 9, we show the the spatial distribution of the (sole) magnetic $\hat{\mathbf{z}}$ component $\left|H_{z}(x, y)\right|$ with respect to the observation point $\left(x / \lambda_{0}, y / \lambda_{0}\right)$, as evaluated by numerical simulations (COMSOL Multiphysics), for three different configurations. In Fig. 9(a) we have copper (metal) alone: an electrically thin slab of copper with thickness $d=0.05 \lambda_{0}$ is excited by the aforementioned magnetic dipole. We can observe the blocking effect of the metallic layer on the incident illumination dictated by image theory. In Fig. 9(b) we have the active counterpart (hosted QDs) alone and one can see the hot spots at the origin and a mild enhancement of the field along the vertical bound- ary reminding of surface plasmon polaritons. In Fig. 9(c), we considered the proposed PT-symmetric structure (two back-toback electrically thin slabs of metal and active medium). The enhancement is huge; much larger than the corresponding of Fig.9(b), despite the fact that an equal amount of activity (i.e. gain contribution of QDs) is used (the one imposed due to the active slab with permittivity $\varepsilon_{2}$ and thickness $d$ ). Most importantly, the field gets concentrated across the separating surface for many wavelengths $\lambda_{0}$ along the $\hat{\mathbf{y}}$ axis which makes this component suitable for wave steering and pattern tailoring.

\section{B. The Case of Kissing Cylinders}

Given the fact that metals are malleable and thus easily shaped into wires, we are examining the configuration of two kissing cylinders [47], [48] one of which is metallic. In particular, two PT-symmetric cylinders (metal of dielectric constant $\varepsilon_{1}-$ left hand side and QDs-doped active medium of dielectric constant $\varepsilon_{2}=\varepsilon_{1}^{*}$ - right hand side) of the same radius $r$ are touching each other. The structure is illuminated by a TM (magnetic field with sole $\hat{\mathbf{z}}$ component) plane wave along an arbitrary angle. The boundary value problem is analytically solvable [49], [50] and the solution can be expanded to cover the anisotropic [51] and the spherical [52] analogous; however, here we confine ourselves to numerical simulations for brevity. This structure has been proposed [47], [48], when both cylinders are metallic, to enhance surface plasmon modes in the vicinity of the common point of the two scatterers. Back in our study, we use a silver cylinder $\left(r=0.3 \lambda_{0}\right)$ as the passive element at $f=600 \mathrm{THz}$ where: $\varepsilon_{1} \cong-7.8-0.78 j$ and a mixture of QDs hosted by a material with permittivity $\varepsilon_{\text {host }} \cong 13$ (semiconductors at the optical frequencies [27], [30], [31], [32]) as the active electrode. The quantum dots should have, according to Figs. 3-7, extremely low losses $(\Gamma \cong 0.0007 f)$ which constitutes a weak point in terms of the system's constructability [33] but, on the other hand, the plasma frequency is ordinary [34]: $f_{P} \cong 0.22 f$. The QDs should get quite easily pumped to the excited state (small $a \cong 0.05)$ and the filling factor is within the permissible limits $(s \cong 40 \%)$.

In Figs. 10, we show the contour plots of the TM magnetic field magnitude in $\mathrm{dB}\left(20 \log \left|H_{z}(x, y)\right|\right)$ on the $\left(x / \lambda_{0}, y / \lambda_{0}\right)$ map, based on numerical simulations, for three different incidence angles. In Fig. 10(a), the system gets excited from the passive side (indicated by the wavy arrow) as in Fig. 9(c). We note that there is an interesting field pattern around the touching point which is comprised of two hot spots and two dark arcs of very low field. The field enhancement is much larger when the structure is illuminated from the active side (Fig. 10(b)), while the relative distribution in the vicinity of the singularity is very similar to that of Fig. 10(a). Such an enhancement is natural because the active sibling is directly fed in the latter case. An intermediate scenario is elaborated in Fig. 10(c), where the plane wave comes from the bottom and the PT-symmetric pairs are excited in a balanced and symmetric way. One observes considerable field confinement and focusing which can enable our structure to operate as accumulator of photonic energy. 


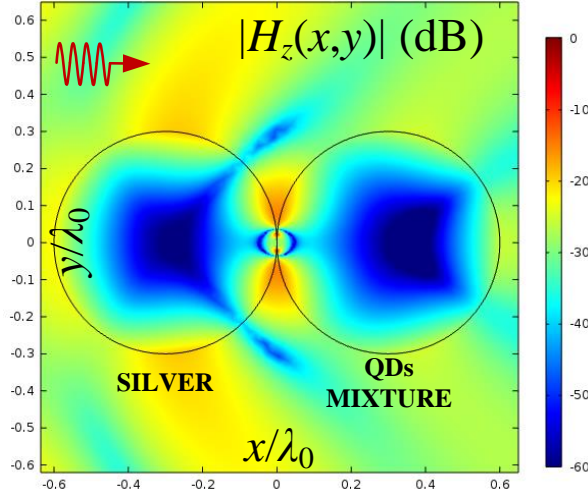

(a)

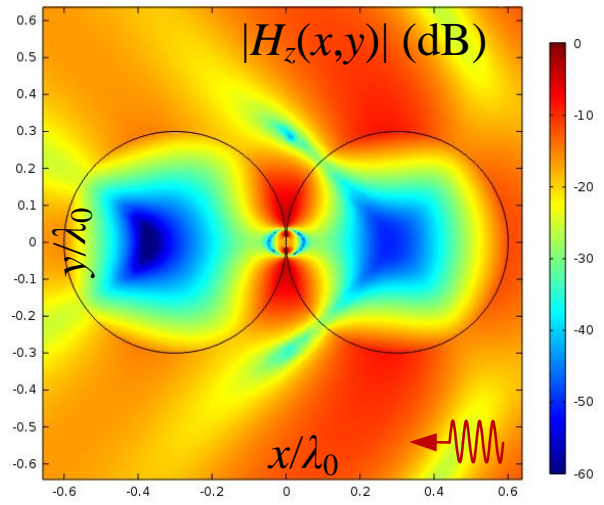

(b)

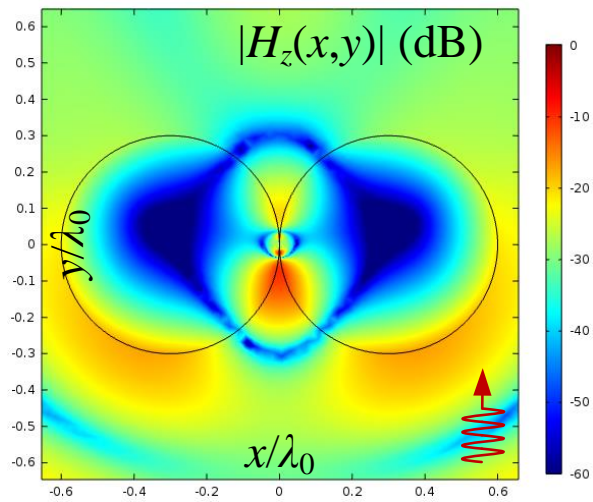

(c)

Fig. 10. The spatial distribution of the magnitude of the magnetic field $20 \log \left|H_{z}(x, y)\right|($ in $\mathrm{dB}$ ) on the $x y$ plane in a configuration of two touching PT-symmetric cylinders of radius $r$ excited by a plane wave coming from: (a) the side of the metal, (b) the side of the active medium, (c) the bottom side. Plot parameters: silver, $f=600 \mathrm{THz}, \varepsilon_{1} \cong-7.8-0.78 j, r=0.3 \lambda_{0}$. The wavy arrows show the direction of plane-wave illumination.

\section{CONCLUSiON}

The characteristics of the frequency-dependent LorentzDrude model of quantum dots when they are hosted by a lossless background medium are optimally determined to provide PT-symmetric siblings for a list of commonly utilized metals at the optical frequencies. These optimal parameters lead to active structures which in certain case are found to be fabricable at least in an approximate sense. The obtained PT-symmetric couples are used to elaborate specific device configurations which are compatible with the metallic texture and have a number of interesting features.

The next step to this study would be to modify the adapted optimization procedure to give multiple candidate mixtures of quantum dots from which the closest to the available real-world active media would be selected. Furthermore, the PT-symmetry objective can get slightly relaxed so that a good result is achieved not at a sole frequency but across a continuous spectral range. We believe that these outcomes, starting from the presented results in this work, may inspire further experimental and theoretical efforts in the emerging field of parity-time engineering of photonic devices.

\section{ACKNOWLEDGMENT}

The author would like to thank the reviewers for their constructive comments.

\section{REFERENCES}

[1] C. M. Bender and S. Boettcher, "Real spectra in non-Hermitian Hamiltonians having PT symmetry", Physical Review Letters, vol. 80, pp. 5243 5246, 1998.

[2] C. M. Bender, S. Boettcher, and P. Meisinger, "PT-Symmetric quantum mechanics", Journal of Mathematical Physics, vol. 40, pp. 2201-2229, 1999.

[3] H. Alaeian and J. A. Dionne, "Non-Hermitian nanophotonic and plasmonic waveguides", Physical Review B, vol. 89, no. 0751362014.

[4] B. Baum, H. Alaeian, and J. Dionne, "A parity-time symmetric coherent plasmonic absorber-amplifier", Journal of Applied Physics, vol. 117, no. 063106,2015

[5] M. Lawrence, N. Xu, X. Zhang, L. Cong, J. Han, W. Zhang, and S. Zhang, "Manifestation of PT Symmetry breaking in polarization space with Terahertz metasurfaces", Physical Review Letters, vol. 113, no. 093901, 2014

[6] H. Benisty, A. Degiron, A. Lupu, A. D. Lustrac, S. Chenais, S. Forget, M. Besbes, G. Barbillon, A. Bruyant, S. Blaize, and G. Lerondel, "Implementation of PT symmetric devices using plasmonics: principle and applications", Optics Express, vol. 19, pp. 18004-18019, 2011.

[7] H. Benisty and M. Besbes, "Confinement and optical properties of the plasmonic inverse-rib waveguide", Journal of the Optical Society of America B, vol. 28, pp. 818-826, 2012.

[8] A. Regensburger, C. Bersch, M.-A. Miri, G. Onishchukov, D. N. Christodoulides, and U. Peschel, "Parity-time synthetic photonic lattices", Nature, vol. 488, pp. 167-171, 2012.

[9] D. L. Sounas, R. Fleury, and A. Alù, "Unidirectional cloaking based on metasurfaces with balanced loss and gain", Physical Review Applied, vol. 4, no. $014005,2015$.

[10] F. Monticone, C. A. Valagiannopoulos, A. Alù, "Aberration-free imaging based on parity-time symmetric nonlocal metasurfaces", http://arxiv.org/abs/1509.07300, Under Review, 2015.

[11] C. A. Valagiannopoulos, F. Monticone, A. Alù, "PT-symmetric planar devices for field transformation and imaging", Accepted for Publication at Journal of Optics, JOPT-102333, 2015.

[12] S. Xiao, V. P. Drachev, A. V. Kildishev, X. Ni, U. K. Chettiar, H.K. Yuan, V. M. Shalaev, "Loss-free and active optical negative-index metamaterials", Nature, vol. 466, pp. 735-740, 2010.

[13] J. A. Gordon and R. W. Ziolkowski "The design and simulated performance of a coated nano-particle laser", Optics Express, vol. 15, pp. 2622-2653, 2007.

[14] I. D. Leon and P. Berini, "Modeling surface plasmon-polariton gain in planar metallic structures", Optics Express, vol. 17, pp. 20191-20202, 2009.

[15] C. Rizza, A. D. Falco, and A. Ciattoni, "Gain assisted nanocomposite multilayers with near zero permittivity modulus at visible frequencies", Applied Physics Letters, vol. 99, no. 221107, 2011.

[16] A. Ciattoni, C. Rizza, and E. Palange, "All-optical active plasmonic devices with memory and power-switching functionalities based on $\varepsilon$-nearzero nonlinear metamaterials", Physical Review A, vol. 83, no. 043813, 2011. 
[17] Thang B. Hoang, G. M. Akselrod, C. Argyropoulos, J. Huang, D. R. Smith, and M. H. Mikkelsen, "Ultrafast spontaneous emission source using plasmonic nanoantennas", Nature Communications, vol. 6, no. 7788, 2015.

[18] P. Tighineanu, M. L. Andersen, A. S. Sorensen, S. Stobbe, and P. Lodahl, "Probing electric and magnetic vacuum fluctuations with quantum dots", Physical Review Letters, vol. 113, no. 043601, 2014.

[19] A. D. Rakic, A. B. Djurisic, J. M. Elazar, and M. L. Majewski, "Optical properties of metallic films for vertical-cavity optoelectronic devices", Applied Optics, vol. 37, pp. 5271-5283, 1998.

[20] Refractive Index Database [Online], refractiveindex.INFO, November 2015.

[21] Y. Fu, L. Thylen, and H. Agren, "A lossless negative dielectric constant from quantum dot exciton polaritons", Nano Letters, vol. 8, pp. 15511555,2008

[22] A. Bratkovsky, E. Ponizovskaya, S.-Y. Wang, P. Holmstrom, L. Thylen, Y. Fu, and H. Agren, "A metal-wire/quantum-dot composite metamaterial with negative $\varepsilon$ and compensated optical loss", Applied Physics Letters, vol. 93, no. 193106, 2008.

[23] K. J. Webb and A. Ludwig, "Semiconductor quantum dot mixture as a lossless negative dielectric constant optical material", Physical Review $B$, vol. 78, no. $153303,2008$.

[24] D. Vanmaekelbergh and L. K. van Vugt, " $\mathrm{ZnO}$ nanowire lasers", Nanoscale, vol. 3, pp. 2783-2783, 2011.

[25] K. Pantzas, A. Itawi, I. Sagnes, G. Patriarche, E. Le Bourhis, A. Lupu, H. Benisty and A. Talneau, "Oxide-free bonding of III-V-based material on silicon and nano-structuration of the hybrid waveguide for advanced optical functions", Photonics, vol. 2, pp. 1054-1064, 2015.

[26] C. A. Valagiannopoulos and A. Sihvola, "Low-pass features of optical nano-filters constituted by simple layered structures", Microwave and Optical Technology Letters, vol. 55, pp. 2099-2106, 2013.

[27] A. Sihvola, Electromagnetic Mixing Formulas and Applications, vol. 47, IEE Electromagnetic Waves Series, IET, ISBN: 0852967721, New York, 1999.

[28] T. G. Mackayab and A. Lakhtakia, "Application of Bruggeman and Maxwell Garnett homogenization formalisms to random composite materials containing dimers", Waves in Random and Complex Media, vol. 25, pp. 429-454, 2015.

[29] P. C. Becker, N. A. Olsson and J. R. Simpson, Erbium-Dopped Fiber Amplifiers: Fundamentals and Technology, Optics and Photonics, Elsevier Academic Press, ISBN: 978-0-12-084590-3, New York, 1999.

[30] M. S. Dresselhaus, Solid State Physics, Lecture Notes, Massachusetts Institute of Technology, Cambridge, Massachusetts, 2001.

[31] F. Wooten, Optical Properties of Solids, Academic Press, ISBN: 1483220761, New York, 1972.

[32] E. D. Palik, Handbook of Optical Constants of Solids Academic, New York, 1985.

[33] E. A. Laird Electrical Control of Quantum Dot Spin Qubits, Ph.D. Dissertation, Harvard University, Cambridge, Massachusetts, 2009.

[34] D. F. Walls and G. J. Milburn, Quantum Optics, Springer Science \& Business Media, New York, 2008.

[35] V. I. Klimov, A. A. Mikhailovsky, S. Xu, A. Malko, J. A. Hollingsworth, C. A. Leatherdale, H.-J. Eisler, and M. G. Bawendi, "Optical gain and stimulated emission in nanocrystal quantum dot", Science, vol. 290, pp. 314-317, 2000.

[36] A. A. Lagatsky, F. M. Bain, C. T. A. Brown, W. Sibbett, D. A. Livshits, G. Erbert and E. U. Rafailov, "Low-loss quantum-dot-based saturable absorber for efficient femtosecond pulse generation", Applied Physics Letters, vol. 91, no. 231111, 2007.

[37] M. T. Harrison, S. V. Kershaw, M. G. Burt, A. L. Rogach, A. Kornowski, A. Eychmuller, and H. Weller, "Colloidal nanocrystals for telecommunications. Complete coverage of the low-loss fiber windows by mercury telluride quantum dots", Pure Applied Chemistry, vol. 72, pp. 295-307, 2000.

[38] V. Axt, M., M. Herbst, and T. Kuhn "Coherent control of phonon quantum beats", Superlattices and Microstructures, vol. 26 pp. 117-128, 1999.

[39] G. Ortner, M. Bayer, Y. Lyanda-Geller, T.L. Reinecke, A. Kress, J.P. Reithmaier, and A. Forchel. "Control of vertically coupled InGaAs/GaAs quantum dots with electric fields", Physical Review Letters, vol. 94, no. $157401,2005$.

[40] A. Laucht, F. Hofbauer, N. Hauke, J. Angele, S. Stobbe, M. Kaniber, G. Bhm, P. Lodahl, M.-C. Amann, and J. J. Finley. "Electrical control of spontaneous emission and strong coupling for a single quantum dot", New Journal of Physics, vol. 11, no. 023034, 2009.
[41] C. E. Ruter, K. G. Makris, R. El-Ganainy, D. N. Christodoulides, M. Segev, and D. Kip "Observation of parity-time symmetry in optics", Nature Physics, vol. 6, pp. 192-195, 2010.

[42] S. Savoia, G. Castaldi, V. Galdi, A. Alù, and N. Engheta, "Tunneling of obliquely incident waves through PT-symmetric epsilon-near-zero bilayers", Physical Review B, vol. 89, no. 085105, 2014.

[43] A. Mostafazadeh, "Spectral singularities and CPA-laser action in a weakly nonlinear PT-symmetric bilayer slab", Studies in Applied Mathematics, vol. 133, no. 353, 2014.

[44] O. V. Shramkova and G. P. Tsironis, "Nonreciprocal scattering by PT-symmetric stack of the layers", 17th International Conference on Transparent Optical Networks (ICTON), 5-9 July 2015, Budapest, 2015.

[45] C. A. Valagiannopoulos, M. S. Mirmoosa, I. S. Nefedov, S. A. Tretyakov, and C. R. Simovski, "Hyperbolic-metamaterial antennas for broadband enhancement of dipole emission to free space", Journal of Applied Physics, vol. 116, no. 163106, 2014.

[46] C. A. Valagiannopoulos, J. Vehmas, C. R. Simovski, S. A. Tretyakov, S. I. Maslovski, "Electromagnetic energy sink", Accepted for publication at Physical Review B, http://arxiv.org/abs/1507.00974, 2015.

[47] Dang Yuan Lei, A. Aubry, S. A Maier, and J. B Pendry, "Broadband nano-focusing of light using kissing nanowires", New Journal of Physics, vol. 12, no. 093030, 2010.

[48] A. Aubry, D. Y. Lei, A. I. Fernandez-Dominguez, Y. Sonnefraud, S. A. Maier, and J. B. Pendry, "Plasmonic light-harvesting devices over the whole visible spectrum", Nano Letters, vol. 10, pp 2574-2579, 2010.

[49] G. O. Olaofe, "Scattering by two cylinders", Radio Science, vol. 5, pp. 1351-1360, 1970.

[50] C. A. Valagiannopoulos, "Electromagnetic scattering from two eccentric metamaterial cylinders with frequency-dependent permittivities differing slightly each other", Progress In Electromagnetics Research B, vol. 3, pp. 23-34, 2008.

[51] C. A. Valagiannopoulos, "Study of an electrically anisotropic cylinder excited magnetically by a straight strip line", Progress In Electromagnetics Research, vol. 73, pp. 297-325, 2007.

[52] C. A. Valagiannopoulos, "Single-series solution to the radiation of loop antenna in the presence of a conducting sphere", Progress In Electromagnetics Research, vol. 71, pp. 277-294, 2007.

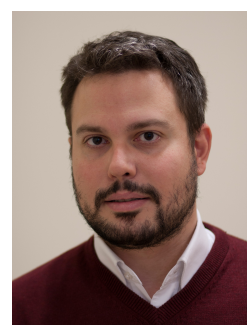

Constantinos Valagiannopoulos was born in Athens, Greece in 1982. He received with honors the Dipl. Eng. degree in Electrical Engineering from National Technical University of Athens (NTUA), Greece in 2004, and the Ph.D. degree for studies on Electromagnetic Theory in 2009. From 2010 to 2015, he worked as a Postdoctoral Researcher in the Department of Radio Science and Engineering of the Aalto University, Finland. During the academic year 2014-2015, he was with the Laboratory of Metamaterials and Plasmonics in the Department of Electrical and Computer Engineering at the University of Texas at Austin, USA. In 2015, he joined Nazarbayev University (NU), Kazakhstan, as an Assistant Professor at Physics Department of the School of Science and Technology.

Dr. Valagiannopoulos's research interests include artificial metamaterials, graphene and their applications in devices controlling the electromagnetic fields (absorbers, cloaks, lenses etc). He has authored or coauthored over 65 works in international refereed scientific journals and presented numerous reports in international scientific conferences.

Dr. Valagiannopoulos is the winner of the inaugural 2015 JOPT research excellence award for his work: Perfect absorption in graphene multilayers. He was also the recipient of the International Chorafas Prize for the Best Doctoral Thesis in 2008 and the Academy of Finland Postdoctoral Grant for 2012-2015. 